\title{
Trends and Directions in Cloud Service Selection
}

\author{
Mona Eisa, Muhammad Younas, Kashinath Basu, Hong Zhu \\ Department of Computing \& Communication Technologies \\ Oxford Brookes University, \\ Oxford, United Kingdom \\ \{mona.eisa-2015, m.younas, kbasu, hzhu\}@brookes.ac.uk
}

\begin{abstract}
With the growing popularity of cloud computing the number of cloud service providers and services have significantly increased. Thus selecting the best cloud services becomes a challenging task for prospective cloud users. The process of selecting cloud services involves various factors such as characteristics and models of cloud services, user requirements and knowledge, and service level agreement (SLA), to name a few. This paper investigates into the cloud service selection tools, techniques and models by taking into account the distinguishing characteristics of cloud services. It also reviews and analyses academic research as well as commercial tools in order to identify their strengths and weaknesses in the cloud services selection process. It proposes a framework in order to improve the cloud service selection by taking into account services capabilities, quality attributes, level of user's knowledge and service level agreements. The paper also envisions various directions for future research.
\end{abstract}

Keywords-Cloud, Service Selection, Quality of Service, Service Level Agreement

\section{INTRODUCTION}

Cloud computing provides flexible, scalable and ondemand IT services over the Internet which has revolutionized the way small, medium and large scale businesses consume IT services. With such a growing demand and popularity of cloud computing, hundreds of technology companies, such as Amazon, Google, Salesforce, Microsoft, IBM, Yahoo, Cloud Provider, Arsys, and many others are trying to capitalize on this emerging market [1]. But there are potential pros and cons of such a large number of service providers. They certainly deliver more choice, better services and savings to consumers (users) who want to move their computing infrastructure into the cloud. However, companies represent the cloud services and selection process based on their own business models and respective service offerings. This makes it confusing for the users to select between different providers. Moreover, users are not always technically competent to estimate and understand their requirements. Therefore, choosing the right cloud services becomes a much harder task to do.

The general procedure for cloud service selection is to have a set of inputs from users and cloud providers about cloud services. Different techniques, methodologies, and algorithms are then used to match the inputs from users with inputs from providers, and to find a best match between them. Users need to identify the quality of service (QoS) attributes they want for their cloud service requirements, providers provide their QoS properties, and the selection tools or techniques compare between the two inputs.

The nature of cloud service selection is different from selecting other online services such as booking a flight or a hotel room. This is due to the distinct characteristics of cloud services. For instance, there are different types of cloud services which are provided through different provisioning models such as SaaS (Software-as-a-Service), PaaS (Platformas-a-Service), and IaaS (Infrastructure-as-a-Service). In terms of selection, SasS services (e.g., Salesforce, Google Apps, and Microsoft Office 365) will have different requirements than PaaS services (e.g., AWS Elastic Beanstalk, Windows Azure, and Heroku) and IaaS services (e.g., Amazon EC2, Rackspace, Cisco Metapod). Moreover, different cloud services have different quality of service attributes such as performance, usability, security, privacy, price and so on. These attributes can be subjective and different users and service providers may have different interpretation and expectation of the quality of cloud services. This problem is further complicated as there is no standard benchmark for measuring the quality of service of cloud services [3].

Recently, various cloud service selection tools and techniques have been emerging as a result of research and development work both in academia and industry. The objectives of commercial tools and the techniques or methodologies designed by researchers are the same. First, these help cloud service providers to market and seamlessly provide their services to potential users. Second, these enable cloud users to find a wide range of cloud services and to choose between cloud service providers that best fit their needs and service demands. Several approaches have been studied by the academia, such as multi-criteria decisionmaking (MCDM) and the multi-criteria optimization technique [2]. Similarly various cloud service selection tools have been developed in the industry such as CLOUDORADO, RankCloudz and Intel Cloud Finder [10].

This paper set out to investigate the current trends and developments in the cloud service selection. It proposes a framework for cloud service selection and also discusses various directions for future research.

The main contributions of this paper are summarized as follows. 
- It presents an integrated cloud service infrastructure that covers the main building blocks of cloud computing and their relation to the cloud service selection. It is used to illustrate the characteristics of cloud services and the consequential intricacies of the cloud service selection.

- It provides a critical review and analysis of the current research work with a focus on the evaluation of three most well-known commercial cloud service selection tools, CLOUDORADO, RankCloudz and Intel Cloud Finder.

- It proposes a new framework for cloud service selection. The main objective of proposed framework is to improve and simplify the service selection process by taking into account user's knowledge and experience of cloud technologies, service level agreements and the wide range of QoS attributes related to cloud services.

- It identifies and discusses various research challenges and sets new directions for future research.

The remainder of the paper is organized as follows. Section II presents a general cloud service infrastructure. Section III explains the main characteristics of cloud and their impact on cloud service selection. Section IV reviews and analyses existing tools and techniques. Section V presents the proposed framework. Conclusion and future research directions are presented in Section VI.

\section{ClOUd SERVICES INFRASTRUCTURE}

In this section we present a general infrastructure for cloud services. It consists of the cloud services, provisioning and deployment models and the service selection procedures at the higher level of abstraction. It is used as a conceptual model to illustrate the characteristics of cloud services and their impact on the service selection procedure. Figure 1 graphically depicts the cloud services infrastructure.

There exist a large number of cloud service providers (or vendors), such as Salesforce, Amazon, Google, Microsoft, Yahoo, Rackspace, Intel, EMC, IBM, Apprenda and many others, which provide users (consumers) with different services such as data storage, software applications, network and communication services, memory/CPU, etc. Cloud services are generally provided in one of the three models:

SaaS (Software-as-a-Service): In it, applications are hosted and run on a cloud infrastructure, but are made available to users over the Internet. Such applications are accessible from various clients such as web browsers or dedicated program interfaces [15]. Examples are Google Apps and Salesforce.

PaaS (Platform-as-a-Service): In PaaS, users are provided with resources required to create applications that are accessible through the internet [14]. Users do not manage or control the underlying infrastructure, but has control over their applications [15]. Examples are Google App Engine. Heroku, Windows Azure.
IaaS (Infrastructure-as-a-Service): It provides users with hardware such as memory, CPU, and storage space in order to deploy and run their applications [14]. Users have control over operating systems, storage, and their applications [15]. Examples are Google Compute Engine, Rackspace, and Amazon EC2.

The most common cloud deployment models are: public cloud, community cloud, private cloud and hybrid cloud.

Public Cloud: In this model cloud infrastructure can be provided to general public so that they can deploy or use their services. Such infrastructure may belong to businesses, academic institutions, or government organization [15].

Community Cloud: In this model, cloud infrastructure is limited for special use by a specific community of users that have shared interests [15]. Healthcare community cloud is a common example of this model.

Private Cloud: In this model an organization or company owns the cloud resources which are used for exclusive use by a single organization [15].

Hybrid Cloud: This model is combination of two or more distinct clouds such as private, community, or public cloud. For example, an organization can use hybrid cloud model where it utilizes public cloud for less-critical applications and private cloud for mission critical applications [13].

Given the diversity of cloud providers and services, cloud provisioning and deployment models, the challenge for users is to select appropriate services that can satisfy their requirements and the terms and conditions of the service providers. In order to deal with this situation various techniques have been developed to enable users to select the right services.

Cloud Service Selection: Cloud service selection is a process in order to discover and select appropriate cloud service providers that can satisfy their requirements based on various constraints such as functional requirements, QoS and Service Level Agreements. The lack of a standardized framework for representation of these requirements makes it difficult to evaluate the quality of services (from different providers) in a way that meet all the requirements from users which abide by the SLAs from service providers. Thus it is important to devise appropriate service selection procedures that evaluate cloud services and select most suitable services.

Service Level Agreement (SLA): SLA is a negotiation mechanism for the contract between the service providers and consumers and the fulfilment of such contract at the run time. In other words, SLA is a legal cloud document that sets the terms and conditions for the usage of services, service data, delivery mode, quality of service, cost/price of services and conditions for penalty in the case SLA is violated [14]. 


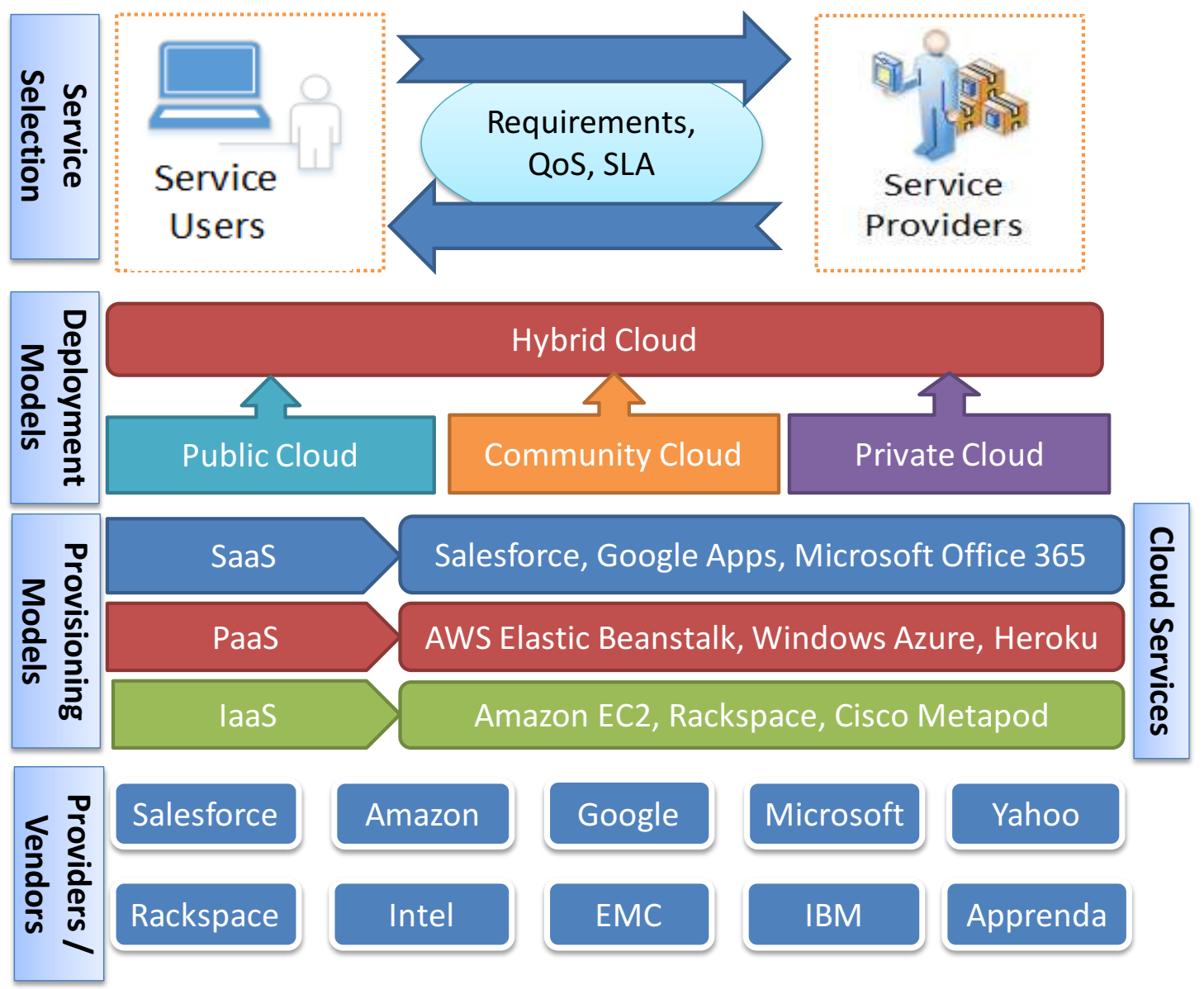

Figure 1. Cloud Services Infrastructure

\section{Characteristics OF Cloud Services}

Compared to classical Internet-based services (e.g., booking a flight or checking weather forecast), cloud services are characterized by distinct features such as elasticity, ondemand service provisioning [15], Service Level Agreement, Quality of Service and so on. Though such features enable a flexible and transparent service provisioning platform they complicate the process of selecting appropriate services that meet user's needs. In the following, we analyse the main characteristics of cloud services and their impact on service selection process.

1. Quality of Service and SLA: Cloud services are associated with a number of QoS attributes such as performance, reliability, security, prices, privacy, usability, and so on. These different QoS attributes make service selection process significantly difficult. For instance, service providers and consumers may have different expectation (or measurement) of QoS attributes. But there is a lack of standard benchmark to measure cloud QoS [3]. Though the SMI is a step forward to standardizing cloud QoS, it still does not provide all the necessary measurements that meet both consumers and providers expectations.

SLA ensures that service providers and consumers abide by the terms and conditions of the contract. For instance, cloud services are provided in a way that meets the QoS requirements. But SLA also makes service selection difficult. Different services providers follow proprietary SLAs and there is a lack of standardization of SLA for cloud services.

2. On-demand service provisioning: Cloud services should be automatically provided in an on-demand self-service style meaning that they require little or no human intervention. But before such automatic provisioning can be ensured service consumers need to correctly specify and understand the technical complexities of the cloud services. For instance, the search and selection service by Intel Cloud Finder can't be used or understood by service consumers unless they have a good knowledge of the cloud technologies.

3. Elasticity: Elasticity is one the essential features of cloud computing wherein services can be elastically provisioned 
and released. On the one hand, this allows consumers to seamlessly acquire or release services based on their needs. On the other hand, this enables providers to allocate and de-allocate resources according to the service availability and demand. However, incorporating elasticity in service selection requires the knowledge of the dynamic changes in service consumption and provisioning and a tool that can keep track of such changes.

4. Resource pooling and distribution: Cloud resources such as storage, CPU, memory, and network bandwidth are pooled by the service providers in such a way that multiple consumers can use such resources using a variety of models such as multi-tenant and virtualization models. Consumers can access resources from the provider pool transparently without knowing the location of the (provided) services. Though such transparency is useful it may give rise to issues such as security, privacy and reliability of services or any other QoS aspects (as described in (1) above). For example, service consumers may raise concerns on the locations of services if they are not reliable and secure.

5. Service provisioning models: Cloud services are generally provided in one of the three models: SaaS (Software-as-aService), PaaS (Platform-as-a-Service), and IaaS (Infrastructure-as-a-Service). These models require service selection tools to enable users that can specify their needs according to the service provisioning models they want to use for getting/using cloud services. For instance, IaaS model may require more low level details (such as memory, CPU, etc) than SaaS which require more high level details about services (such as speed, usability, etc).

6. Service deployment models: The most common cloud deployment models are: public cloud, community cloud, private cloud and hybrid cloud. Public cloud offers greater flexibility in terms of service provisioning but it also requires greater level of security and privacy. Private cloud is considered more secure but is limited to service provisioning over a private network and a private hosting. Thus cloud service selection tools should be designed in a way that caters for the required level of QoS and SLAs of the different deployment models.

\section{Cloud Service Selection ToOls And Techniques}

Academic work on cloud service selection has been thoroughly reviewed $[2,11]$. But commercial cloud service selection tools have not been reviewed nor analysed in the existing literature. This section first reviews some of the existing academic works which is followed by a comparative evaluation of the commercial tools.

\subsection{Analysis of Academic Work}

Various models and techniques have been proposed and developed for cloud services selection in academic research $[2,11]$.

One of the trends is to exploit Service-Oriented Architecture (SOA) in cloud service selection and provisioning. This is due to the fact that cloud services are provisioned using the Web and Internet technologies. SOA has been developed for the delivery of web services over the Internet. SOA also enables the cloud to be delivered in the form of services to the end users using the Web and Internet. The service users can tailor the services they want to match their requirements and the service providers can provision a service that meets user's requirement $[8,9]$.

Sun et al. [2] reviewed a number of cloud service selection models and techniques. The existing techniques were first classified into two main categories: multi-criteria decisionmaking (MCDM) method and multi-criteria optimization method. Each of the methods and techniques was then analyzed in terms of the inputs and outputs of the methods, the application context, the different parameters needed, and the language or model used. Based on the review and analysis, some interesting open challenges were identified for further research. These include the lack of cloud marketplace and the lack of advanced measurement of user preferences.

Manvi et al. [11] surveyed various techniques that address the research management issues. They used metrics to quantify each of schemes. The authors claimed that the challenge for resource management is to determine the resource demand of each application and to allocate resource in a most efficient way. This survey gives an insight into the complexity of resources management. It also helps in understanding how the management of resources can enhance service selection, optimization and simplification.

Baranwal et al. in [3] proposed a cloud service selection model using ranked voting. In this method a normalized preference score is calculated for each cloud provider. Provider with the highest normalized preference score is the winner and is selected as the best cloud provider.

In cloud service selection, one of the most important factors is the cloud service quality attributes that help users express their requirements. Quality of Service (QoS) attributes are most commonly used in the cloud service selection process. Burkon [6] proposed a set of QoS attributes that are used in cloud service selection process, especially for SaaS model. In [7], CSMIC introduced the Service Measurement Index (SMI). SMI is a hierarchical framework with seven categories. Each category is further refined by four or more attributes [7]. The objective is to standardize Key Performance Indicator (KPI) for measuring and comparing services.

Service Level Agreement (SLA) is another crucial element of the cloud services. Baset [12] reported that each cloud service is typically accompanied by an SLA which defines minimal guarantees that a provider offers to its customers. However, there is no standardized SLA and different providers use their proprietary SLAs. This makes it difficult for cloud users to compare different SLAs and consequently the selection of cloud services. The work in [12] advocated a standard cloud SLA which should typically have the following main components: service guarantee, service guarantee time period, service guarantee granularity, service guarantee 
exclusions, service credit, and service violation measurement and reporting. The definition of such components would help to devise standardized SLAs.

In summary, we make the following observations of the current research and their pros and cons in relation to the cloud service selection.

- The idea of standardising SLAs would be appealing to both cloud users and providers. Cloud brokers, mediating between users and providers, would also benefit from a standardized SLA. However, the large number of cloud providers and users, the different types of services and locations (different countries and places) pose a big hurdle to develop standardised SLAs.

- The Service Measurement Index (SMI) is a useful step towards standardising the quality attributes or the Key Performance Indicator (KPI) for measuring and comparing services. But some of the quality attributes can be quite subjective and their measurements can be perceived differently by different people (users or providers). For instance, the price of the same service under same conditions can be perceived as cheap by some while as expensive by the others.

- Though current cloud service selection techniques provide some useful solutions most of them appear to be too complex for ordinary users. Users with limited or no technical knowledge of cloud technologies would find it extremely difficult to use existing solutions.

- Current techniques do not provide uniform representation and definition of the quality attributes. Different providers use different terminologies to define quality attributes for the services they offer to users. This also makes service selection difficult.

\subsection{Analysis of Commercial Tools}

Recently commercial search and selection tools for cloud services are emerging in order to help users choose the right services that meet their requirements. Here, we only focus on the three well known tools, which are Intel Cloud Finder ${ }^{1}$, CLOUDORADO $^{2}$, and RankCloudz ${ }^{3}$.

Due to the commercial nature of these tools we were not able to get hold of the underlying models, algorithms or other technical details related to the design and development of these tools. We therefore compare these tools based on the information provided through their web interfaces (web pages) which are publicly available on the Internet.

Intel Cloud Finder: It offers two ways of searching and choosing cloud providers: Quick Search and Detailed Search. The Quick Search allows users to narrow down the list and quickly choose a cloud provider. Users can select different criteria as shown in Figure 2. Users can quickly choose a provider by selecting attributes that match the offerings of a

\footnotetext{
${ }^{1} \mathrm{https}: / / \mathrm{www}$. intelcloudfinder.com/

${ }^{2}$ https://www.cloudorado.com/

${ }^{3}$ https://www.rightcloudz.com/RankCloudz
}

cloud provider. The tool dynamically changes the list of providers presented to the user depending on the user's choice of attributes. Some of attributes are briefly explained in order to help user understand them. For example, the "Pay-as-YouGo" attribute is defined as "There is a set fixed price for a particular instance type which is charged at an hourly rate until the instance is terminated".

The Detailed Search tool gives more flexibility to the users. Users are presented with six main parameters, each of which has sub-parameters that contain features users might find them important. Users can specify rating for a particular feature as "Essential", "Desirable", "Future" or "N/A". Users also have the option of prioritizing one feature over another. Based on the search criteria results provided by the tool gives some sort of ranking to the cloud providers depending on the importance of the features the user has selected.

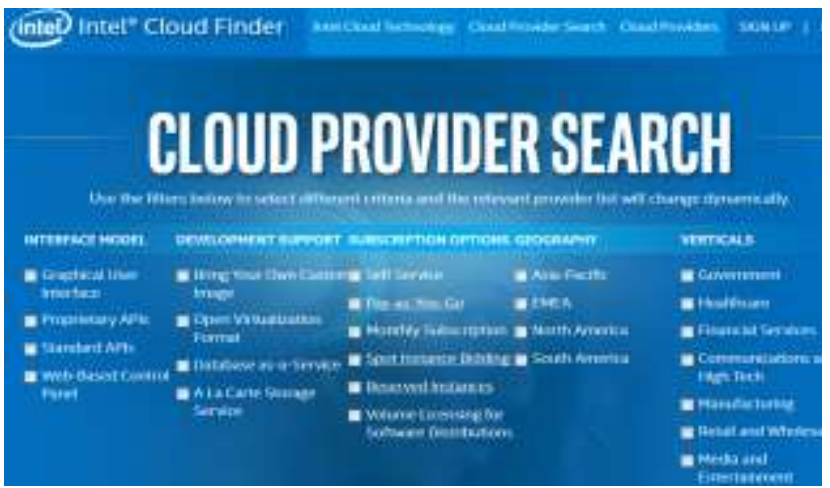

Figure 2. Intel Cloud Finder

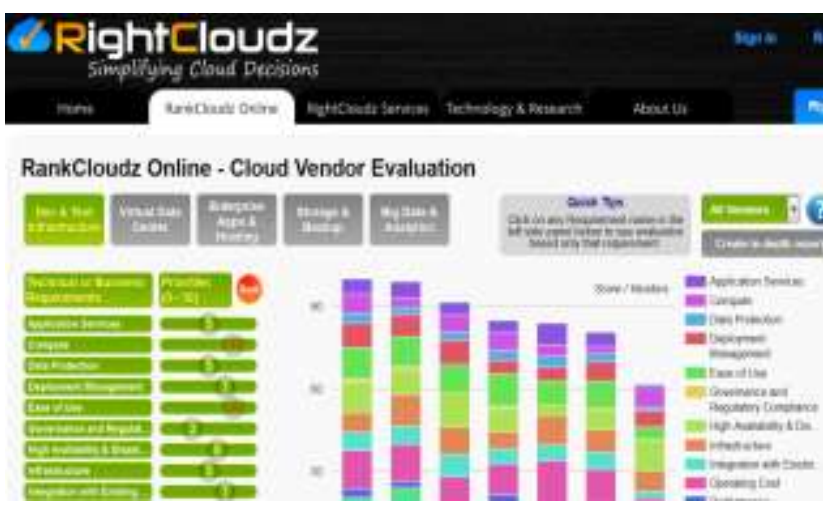

Figure 3. RankCloudz

RankCloudz: Similar to Intel Cloud Finder's detailed search, RankCloudz allows users to give importance to a particular feature by setting its priority. As shown in Figure 3, RankCloudz divides the Cloud Computing platforms into five different scenarios: Dev \& Test Infrastructure, Virtual Data Center, Enterprise Apps \& Hosting, Storage \& Backup and Big Data \& Analytics. Each of the categories has their own parameters and user can configure their priority. Priority is assigned on scale from 0 to 10 , where 0 is the lowest and 10 is the highest priority. RankCloudz ranks the cloud providers 
depending on the parameters and their priorities (set by the user). A dynamic graphical representation of rankings with color-coded parameters is presented to the user. An in-depth report is also available to the user which offers description of the parameters as well as the ranking values that had been generated for each of them depending on their priority.

Cloudorado: As shown in Figure 4, Cloudorado divides the Cloud Computing into three categories; Cloud Server, Cloud Hosting and Cloud Storage. It has also a dedicated page that presents list of cloud providers, their offered features and parameters in a tabular form. Cloudorado performs similar to quick search of Intel Cloud Finder, but unlike Intel it does not offer advanced search. Users can select the parameters that satisfy their requirements and a dynamic list is produced based on the user requirements. Cloudorado offers brief description of the parameter in the form of a Tooltip. The result is sorted by price with the lowest cost displayed near to the top. Compared to RankCloudz and Intel Cloud Finder, Cloudorado does not produce a comprehensive result. It only offers users link to the Cloud Provider's website.

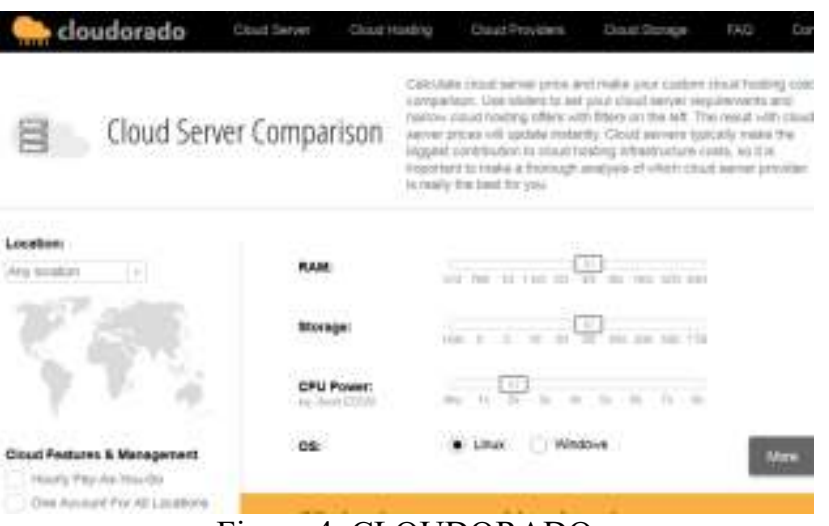

Figure 4. CLOUDORADO

We use the following criteria to analyse and compare these tools.

1. Usability: This is to evaluate the general usage of the tool. That is, how easy it is to use the tool keeping in mind the user's knowledge as well as information provided for each of the providers in the list.

2. Understanding of QoS parameters (attributes): Cloud services have many components that can be configured by the user. Therefore, QoS parameters (e.g., performance, reliability, security, etc.) presented to the users need to have detailed explanation on their use especially for nontechnical users.

3. Understanding of service description: Similar to the above, service descriptions (e.g., memory, storage space, network bandwidth, protocol uses, etc.) presented to the users need to have detailed explanation on their use especially for non-technical users.
4. Support for selecting service provisioning models: Do the selection tools enable users to select services based on IaaS, PaaS, and SaaS models?

5. Support for selecting deployment models: Do the selection tools take into account the deployment models such as Public, Community, Private and Hybrid clouds?

6. Number of cloud providers: How many cloud providers are listed in the database/repository of a selection tool? Having more providers in a database would provide more options for users to choose from.

7. Data about services: How do selection tool collects data or information about the providers and their services. Is the data collected dynamically and in real time or is it collected statically?

8. Evaluation of services: Do the tools provide evaluation report of the services selected by the users?

9. Advanced search: Do the tools provide advanced search or basic search facilities?

10. User guide (manual): Is there a user guide or a manual that can help users to understand the use of the tool and the results it returns to the users?

11. Visualization of results: How are the results presented to the users? Presenting results in a complex way can push users away from using the tool?

12. SLAs integration: Do the tools integrate SLAs with the search and selection process?

Table 1 provides a summary of the analysis of the three tools.

Usability (ease of use) is one of the important features of the service selection tools. As shown in Table 1, the usability of RankCloudz is set as 'high'. Out of the three tools, RankCloudz is easiest to use because user is presented with 11 to 13 attributes. User has to set priority for each attribute on a scale of $0-10$ (0 is lowest and 10 is highest). To make it even simpler, the result is presented next to the scale so that users can see the result as soon as they set values for quality attributes. The result is shown in a graphical representation of the cloud providers ranked with colour coded parameters.

In terms of 'Understanding of the quality attributes' none of the tools provides an easy-to-understand description for the quality attributes. But Intel Cloud Finder, especially in the Detailed Search option, provides some description for each quality attribute. User is presented with six main parameters, all of which have sub-parameters that contain features users might find important. The six parameters are security, usability, quality, availability, technology, and business. However, some of the descriptions are rather confusing and are not clearly linked to the actual parameters. RankCloudz also provides description for each of the attributes, but user needs to download an in-depth report to be able to find the description. Descriptions of attributes are not presented on the webpage of the tool. Users have to go through several steps to find the descriptions. They have to sign up or log in to the tool, set parameters for cloud provider selection, download the 
in-depth report, open the in-depth report with PDF viewer, and then they would be able to access the descriptions.

In terms of 'Understanding of service description' Cloudorado appears to be better than the other two. It offers a brief description of the parameters in the form of a tooltip. When a user hovers the mouse over the parameter's name, a tooltip with a brief description of the parameter is presented to the user.

As shown in the table, all the three tools support only IaaS services such as selection of memory, disk storage, CPU, etc. $\mathrm{PaaS}$ and SasS-based services are not included yet. Cloudrado has indicated that they plan to include PaaS services in future.

None of tools indicate any support for cloud deployment models. This is marked as not applicable (N/A) in the table as it may not be necessary to identify the deployment model during the selection process.

Intel Cloud Finder has the highest number of cloud providers followed by the Cloudrado. RankCloudz does not specify the number of providers. Thus Intel would provide more choices of services for the users to choose from.

All of the three tools keep only static data about the providers and their services. They do not get dynamic updates of any changes to the cloud services.

RankCloudz provides an in-depth report which includes evaluation methodology, short summary of the evaluation results, strengths and limitation about the recommended cloud providers. Intel Cloud Finder provides evaluation report but is rather basic. Cloudrado does not provide any such report.

Intel Cloud Finder offers two types of searches: Quick and Detailed. Cloudrado offers three different categories for users to choose from. These include, cloud server, cloud hosting, cloud providers and cloud storage. RankCloudz offers five different options for the users to choose from based on their needs: Development \& Test Infrastructure, Virtual Data Centre, Enterprise APPS \& Hosting, Storage \& Backup and Big data \& Analytics.

Intel Cloud Finder and Cloudrado do not provide a User Guide. But RankCloudz provides a User Guide explaining how to use the tool.

The three tools present the results differently. These are explained as follows.

Intel Cloud Finder's quick search option generates a tabular list of cloud providers based on user's selection. On clicking any provider, it redirects users to the respective cloud provider's websites. The detailed search produces a table of the parameters, user choices $(\mathrm{E}, \mathrm{D}, \mathrm{F}, \mathrm{N} / \mathrm{A})$ and the top providers matching user's requirements as column headers. It shows results (as percentages) about the cloud providers with respect to the user's choices.

Cloudrado produces a list of cloud providers depending on the parameters selected. The list is sorted according to the prices. The list provides links to the providers' websites.
RankCloudz produces interactive stacked bar charts of the different parameters where each parameter is colour coded. The length of the bar for a single parameter is dependent on the score it gets. Each parameter has a score dependant on its priority plus other factors. But RankCloudz website does not give any details of such factors. The calculation of the scores is not publicly available. It provides a filtering mechanism with ranking involved.

Finally, none of the tools integrates the SLA into the service selection process.

Table 1: Summary of the analysis of commercial tools

\begin{tabular}{|c|c|c|c|}
\hline Criteria & $\begin{array}{c}\text { Cloud } \\
\text { Finder }\end{array}$ & $\begin{array}{c}\text { Cloud- } \\
\text { rado }\end{array}$ & $\begin{array}{c}\text { Rank- } \\
\text { Cloudz }\end{array}$ \\
\hline Usability & Low & Low & High \\
\hline $\begin{array}{c}\text { Understanding of quality } \\
\text { attributes }\end{array}$ & Fair & Low & Low \\
\hline $\begin{array}{c}\text { Understanding of service } \\
\text { description }\end{array}$ & Low & Fair & Low \\
\hline Selection of IaaS services & Yes & Yes & Yes \\
\hline Selection of PaaS services & No & Maybe & No \\
\hline Selection of SaaS services & No & No & No \\
\hline Cloud deployment models & N/A & N/A & N/A \\
\hline Number of cloud providers & 98 & 25 & Unclear \\
\hline Data about services & Static & Static & Static \\
\hline Evaluation report & Basic & No & Detail \\
\hline Advanced search & Yes & Yes & Yes \\
\hline User guide (manual) & No & No & Yes \\
\hline Visualization of results & Grid & Sorted & $\begin{array}{c}\text { Bar } \\
\text { list }\end{array}$ \\
\hline SLAsts integration & No & No & No \\
\hline
\end{tabular}

In summary, commercial cloud selection tools facilitate the selection process to some extent. But there still exist areas for further enhancements such as improving usability, accounting for user knowledge and experience, description of QoS attributes, and incorporation of SLA.

\section{THE PROPOSED FRAMEWORK}

This section presents the framework we propose to address some of the challenges in the cloud service selection. It is still under development. We therefore illustrate the conceptual design and its potential contributions.

The conceptual design of the proposed framework is shown in Figure 5. It is composed of various building blocks (or component modules), which include:

Cloud Providers Knowledge Repository: Cloud Providers Knowledge Repository is the main knowledge storage of the 
proposed framework. This repository stores all information related to cloud services offered by each provider registered in the repository.

One of the main goals of the proposed framework is to provide most credible information about the services and their quality attributes to users when they are selecting cloud services. It therefore exploits the following three component modules in order to ensure the credibility (or correctness) about the services provided by the cloud services. These include:

Provider Scrapper: This module is to provide relevant APIs that can connect to different cloud providers' websites and to extract up to date information about the services, their quality attributes and SLA. Provider scraper will constantly check cloud providers websites to see if there is any new information such as new pricing, new service offering, or new security standards. When the provider scraper finds new information from a cloud service provider, it will store such information into the Knowledge Repository as shown in Figure 5. This information will then be used by the service selection algorithm.

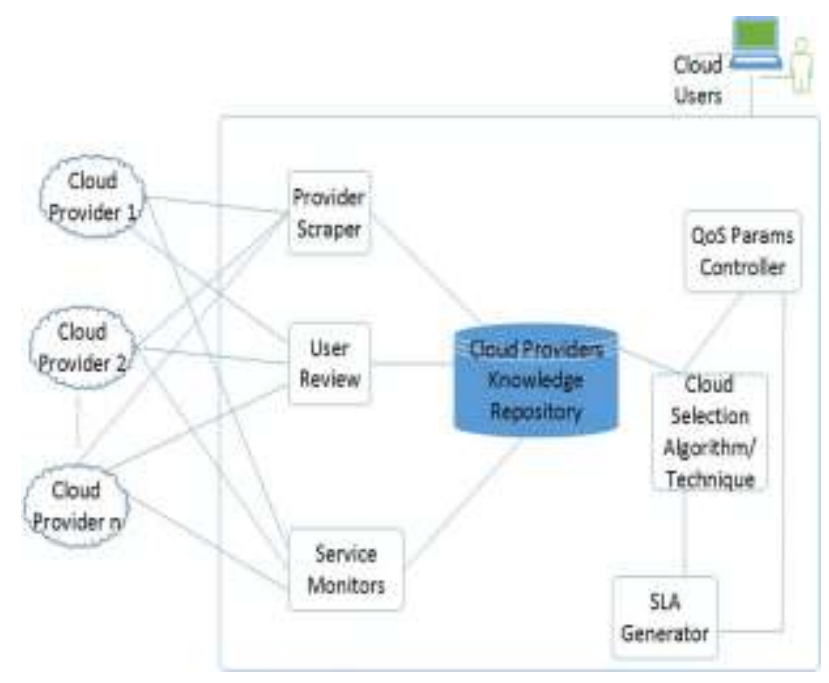

Figure 5. Architecture of the Proposed Framework

User Reviews: This module is to extract and manage information about user reviews of the cloud services. Similarly to other online services (e.g., flight booking or utility services) cloud users should be able to provide reviews for cloud services. This information can then be used in order to help other users in selecting cloud services. The User Reviews module will retrieve required information and store them into the Knowledge Repository.

Service Monitors: Its main responsibility is to monitor the latest status of cloud services from cloud providers. Our intention is not to develop new monitoring tools but rather to exploit existing tools (e.g., cloudharmony) which regularly check the performance and reliability of services of cloud providers. This data can then be stored in Knowledge Repository and be utilised in the service selection process. However, it may not be possible that all cloud services can be monitored as some of them may not have interfaces to the monitoring tools.

Cloud Service Selection: This will implement the new service selection algorithms in order to filter and select appropriate cloud services that can potentially meet cloud users' requirements. This is one of the main components of the proposed framework.

It will take the input from QoS Parameters Controller module, as well as cloud service information received from the Knowledge Repository. In order to ensure the credibility (and correctness) of the service selection, this module will utilise information received from the above three different sources (or components) such as Provider Scrapper, User Reviews, and Service Monitors.

This module will then apply selected algorithms to compare user requirements against the aggregated service information. This module will produce a shortlist of best-fit providers and services. This shortlist will then be presented to the users.

QoS Parameters Controller: QoS Parameters Controller module's main responsibility is to enable users for specifying QoS requirements of the services and to process users inputs. The user requirements of the required QoS are then fed into the selection algorithms of Cloud Service Selection (as described above). This module will also represent QoS parameters in a uniform way so that users with little knowledge (of cloud technologies) can easily understand and specify their requirements.

SLA Generator: SLA Generator module's main responsibility is to generate SLA based on user's input of the QoS requirements. That is, SLA terms and conditions are to be linked to the specified QoS parameters. This is to enable users to understand (or know) the associated SLAs when selecting cloud services.

In summary, the objectives and potential contributions of the proposed framework are to help cloud users in selecting the best cloud services that fit their needs.

- Unlike existing solutions, the proposed framework takes into account the level of user's knowledge in relation to the (technical and complex) descriptions and definitions of the cloud services and the quality attributes. While existing cloud selection tools present overwhelming quality attributes to users to choose from, this framework would present a set of attributes that are tailored according to users knowledge and experience of the cloud computing. For instance, cloud users with a minimum knowledge of the technicality of cloud computing will be provided with a simplified set of attributes compared to cloud users who have more knowledge or experience of the cloud computing. 
- Service Level Agreement (SLA) is one of the key components in cloud service selection and provisioning. Even after users find the best cloud services that fit their needs and requirements, they still need to agree to the SLA which specifies the terms and conditions for the usage of services. The proposed framework will integrate the SLA with the quality attributes so that users can get instant (on the fly) SLA information when selecting cloud services. This would enable users to select services based on the quality attributes and the associated terms and conditions of the SLA. It is possible that users select best services based on their quality attributes but they may not agree to the terms and conditions of the SLA if they are difficult (or expensive) to meet.

- Online users' reviews are increasingly playing an important role in the selection of online (web-based) services such as booking a flight or a hotel, buying a mobile phone, and contracting to utility services such as gas and electricity. We believe that incorporating users reviews in the cloud service selection would significantly help users in selecting cloud services. In addition, such reviews (or feedback) would help cloud providers to improve their services based on user experience of their services. Enabling cloud providers to see how their services perform from users' point of view, would help in their decision making process. On the contrary, existing cloud service selection tools and techniques provide no facility for cloud users to give feedback or review of cloud services.

- In order to ensure the credibility (and correctness) of the service selection, the proposed framework utilise information received from the three different sources such as Provider Scrapper, User Reviews, and Service Monitors. This is believed to provide unbiased rating of cloud services. Existing solutions generally make use of a single source of information, which is susceptible to incorrectness or inconsistency.

\section{CONCLUSION AND FUtURE RESEARCH}

This paper investigated into the challenging issue of the cloud service selection. Such issue has become critical given the large number of cloud service providers, the different types of services and their QoS and SLA requirements. Thus choosing the right cloud services has become a challenging task for cloud users.

This paper presented an integrated cloud infrastructure in order to illustrate the characteristics of cloud services and the consequential intricacies of the cloud service selection. It provided a critical review of the different solutions including research and commercial cloud service selection tools. Motivated by the current issues the paper also proposed a new framework for cloud service selection which aims to improve and simplify the service selection process.

We perceive that the maturity of service selection solutions should not only improve and simplify service selection process but it should also open new directions in cloud computing research. Some of these are described as follows.
- Service switching or migration: Service selection tools and techniques can enable transparent and seamless migration of services from one cloud provider to another. For instance, uSwitch (http://www.uswitch.com/) provides an online comparison and switching service in order to help users to compare utility service prices and products such as gas, electricity, phone, etc., from different suppliers. Based on the recommendation from uSwitch, customers can then switch their services from one supplier to another. In future, cloud tools and techniques should be developed so that cloud users can easily switch their services from one provider to another. However, current solutions do not provide an easy switching or migration of services. The absence of facilities for service switching is also one of the main reasons for lock-in problem.

- Cloud vendor lock-in: The problem of vendor lock-in is one of the major obstacles in cloud service adoption. Due to the complexities of cloud services many users stay with existing cloud providers even though they may not meet their needs. Similar to utility services (like gas, electricity, phone) cloud users should not be locked-in with one vendor. Instead, there should be tools, techniques and methods in place, the help solve the vendor lock-in problem.

- Cloud brokers: Design and development of cloud broker services can assist and advice cloud users and providers on the selection and provisioning of cloud services. Cloud brokers will act as an intermediary between cloud providers and cloud users. Broker services can benefit from cloud service selection tools as they can recommend appropriate services to the users.

\section{REFERENCES}

[1] Elsenpeter RC, Velte TJ and Velte AT (2009) Cloud Computing: A Practical Approach (1st edition). New York: McGraw-Hill Professional Publishing

[2] Sun, L., Dong, H., Hussain, F. K., Hussain, O. K. and Chang, E. (2014) 'Cloud service selection: State-of-the-art and future research directions', Journal of Network and Computer Applications, 45, pp. 134-150. doi: 10.1016/j.jnca.2014.07.019

[3] Baranwal, G. and Vidyarthi, D. P. (2016) 'A cloud service selection model using improved ranked voting method', Concurrency and Computation: Practice and Experience, , p. n/a-n/a. doi: 10.1002/cpe. 3740 .

[4] Garg, S.K.; Versteeg, S.; Buyya, R., "SMICloud: A Framework for Comparing and Ranking Cloud Services," in Utility and Cloud Computing (UCC), 2011 Fourth IEEE International Conference on , vol., no., pp.210-218, 5-8 Dec. 2011. doi: 10.1109/UCC.2011.36

[5] Zibin Zheng; Xinmiao Wu; Yilei Zhang; Lyu, M.R.; Jianmin Wang, "QoS Ranking Prediction for Cloud Services," in Parallel and Distributed Systems, IEEE Transactions on , vol.24, no.6, pp.1213-1222, June 2013. doi: 10.1109/TPDS.2012.285

[6] Burkon, Lukas, "Quality of Service Attributes for Software as a Service", Journal of System Integration, vol. 4 issue 3, pp. 38, September 2013

[7] "Service Measurement Index Framework Version 2.1", July 2014, Carnegie Mellon University Silicon Valley

[8] Duan Q (2011) Modeling and Performance Analysis on Network Virtualization for Composite Network-Cloud Service Provisioning. 2011 IEEE World Congress on Services.

[9] Yu R, Yang X, Huang J, Duan Q, Yan and Tanaka Y (2012) QoS-aware service selection in virtualization-based Cloud computing. 2012 14th 
Asia-Pacific Network Operations and Management Symposium (APNOMS).

[10] Intel Corporation (2012) Intel® Cloud Finder - Cloud Service Providers Search Tool. [Online] Available at: http://www.intelcloudfinder.com/ (accessed 03/06/15).

[11] Manvi SS and Shyam GK (2014) Resource management for Infrastructure as a Service (IaaS) in cloud computing: A survey. Journal of Network and Computer Applications. 41, 424-440.

[12] Baset, S. A. (2012) 'Cloud SLAs: Present and Future', ACM SIGOPS Operating Systems Review, 46(2), p. 57. doi: 10.1145/2331576.2331586.

[13] B.O. Apduhan, M. Younas, T. Uchibayashi (2015) "Improving Reliability and Availability of IaaS Services in Hybrid Clouds", The 15th International Conference on Computational Science and its
Applications (ICCSA 2015), June 22-25, 2015, Banff, AB, Canada, LNCS 9155, Springer 2015, ISBN 978-3-319-21403-0

[14] K. Pande Joshi and C. Pearce (2015) "Automating Cloud Service Level Agreements using Semantic Technologies", Proc. of the 2015 IEEE International Conference on Cloud Engineering (IC2E), 9-13 March 2015, Tempe, AZ, USA, pp.416-421

[15] Velte, A.T., Velte, T.J. and Elsenpeter, R.C. (2009) Cloud computing: A practical approach. New York: McGraw-Hill Professional Publishing.

[16] Mell, P. and Grance, T. (2012) The NIST definition of cloud computing recommendations of the national institute of standards and technology special publication 800-145. Available at: http://nvlpubs.nist.gov/nistpubs/Legacy/SP/nistspecialpublication800 145.pdf (Accessed: 8 February 2016). 